\title{
FAM172A protein promotes the proliferation of human papillary thyroid carcinoma cells via the $\mathrm{p38}$ mitogen-activated protein kinase pathway
}

\author{
MEI-FANG LI ${ }^{1,2^{*}}$, RONG ZHANG ${ }^{1,2^{*}}$, MING-GAO GUO ${ }^{3 *}$, LIAN-XI LI $^{1,2}$, \\ HAN-KUI LU ${ }^{4}$, JUN-XI LU ${ }^{1,2}$ and WEI-PING JIA ${ }^{1,2}$
}

\author{
${ }^{1}$ Department of Endocrinology and Metabolism, Shanghai Jiao Tong University Affiliated Sixth People's Hospital; \\ ${ }^{2}$ Shanghai Diabetes Institute, Shanghai Clinical Center for Diabetes, Shanghai Key Clinical Center for Metabolic Diseases, \\ Shanghai Key Laboratory of Diabetes Mellitus; Departments of ${ }^{3}$ Surgery and ${ }^{4}$ Nuclear Medicine, \\ Shanghai Jiao Tong University Affiliated Sixth People's Hospital, Shanghai 200233, P.R. China
}

Received December 30, 2014; Accepted October 19, 2015

DOI: $10.3892 / \mathrm{mmr} .2015 .4548$

\begin{abstract}
Family with sequence similarity 172, member A (FAM172A), was cloned from human aortic tissues and confirmed in our previous study in 2009, however, its functions remain to be fully elucidated. In our previous studies, the protein expression of FAM172A in human aortic smooth muscle cells was found to be upregulated by high glucose in a concentration- and time-dependent manner. Several reports have shown that insulin resistance is associated with papillary thyroid carcinoma (PTC). Thus, in the present study, the protein expression levels of FAM172A in human papillary thyroid carcinoma were investigated, and the effect of the FAM172A protein on the proliferation of $\mathrm{IHH}-4$ human papillary thyroid carcinoma cells, and its potential molecular underlying mechanisms were examined. Immunohistochemistry and western blotting demonstrated that the protein expression of FAM172A in papillary thyroid carcinoma tissues was not only significantly higher than that in noncancerous tissues adjacent to the carcinoma tissues, but it was also markedly higher than that in normal thyroid and thyroid adenoma tissues. Overexpression of the FAM172A protein activated the p38 MAPK pathway, but not the PI3K and AMPK pathways, in the IHH-4 cells. In addition, overexpression of the FAM172A protein accelerated IHH- 4 cell proliferation, compared with the control group, and the pro-proliferative effect of FAM172A protein on IHH4
\end{abstract}

Correspondence to: Dr Lian-Xi Li, Department of Endocrinology and Metabolism, Shanghai Jiao Tong University Affiliated Sixth People's Hospital, 600 Yishan Road, Shanghai 200233, P.R. China E-mail: lilx@sjtu.edu.cn

\section{*Contributed equally}

Key words: family with sequence similarity 172 member A, papillary thyroid carcinoma, cell proliferation, p38 mitogenactivated protein kinase cells was markedly attenuated by SB202190, an inhibitor of p38 MAPK. Taken together, these results suggest that the FAM172A protein is expressed at high levels in human PTC, which may promote cell proliferation via activation of the $\mathrm{p} 38$ MAPK signaling pathway, and be involved in the pathogenesis of PTC.

\section{Introduction}

Family with sequence similarity 172, member A (FAM172A), also termed C5orf21, was first cloned from human aortic tissues and its existence was confirmed in our previous study in 2009 (1,2), however, its biological function and role in disease states remains to be fully elucidated. In our previous studies, the protein expression of FAM172A was observed in human endothelial cells, vascular smooth muscle cells and macrophages, and was upregulated by high glucose in a concentration- and time-dependent manner $(2,3)$, which indicated that FAM172A may be involved in the pathogenesis of diabetic macroangiopathy.

Previously, Harding et al (4) reported that type 1 and type 2 diabetes are associated with increased incidence and mortality rates from cancer. In addition, Ayturk et al (5) indicated that insulin resistance is an independent risk factor for thyroid nodule formation, and several studies reported that insulin resistance was markedly associated with the occurrence of papillary thyroid carcinoma (PTC) (6-8), accounting for $\sim 80-85 \%$ of all cases of thyroid cancer $(9,10)$.

Whether the FAM172A protein also has positive effects on PTC remains to be fully elucidated. Therefore, the present study aimed to first investigate the protein expression levels of FAM172A in human PTC, followed by the examination of the effect of the FAM172A protein on the proliferation of $\mathrm{IHH}-4$ human PTC cells and its potential molecular mechanisms.

\section{Materials and methods}

Human thyroid tissues. A total of 12 thyroid specimens wre collected from nine patients (age, 34-67 years; 7 female 
patients, 2 male patients) with thyroid diseases, including three normal thyroid, three thyroid adenoma, three PTC and three PTC corresponding pericarcinous tissue samples were collected from patients undergoing thyroidectomy in the Department of Surgery, Shanghai Jiao Tong University Affiliated Sixth People's Hospital (Shanghai, China) between July 2012 and January 2013, and were stored at $-80^{\circ} \mathrm{C}$. The present study was approved by the human research ethics committee of Shanghai Jiao Tong University Affiliated Sixth People's Hospital, and written informed consent was obtained from all participants prior to commencement.

Immunohistochemical staining. The thyroid specimens were fixed with $4 \%$ paraformaldehyde (Shanghai Ling Feng Chemical Reagent Co., Ltd., Shanghai, China) in ice-cold phosphate-buffered saline (PBS) for $10 \mathrm{~min}$, and rinsed three times in PBS, followed by incubation with $20 \%$ sucrose overnight. Subsequently, the frozen tissue samples were sectioned $(4 \mu \mathrm{m})$ and mounted on polylysine pre-coated glass slides with paraformaldehyde solution with $3 \% \mathrm{H}_{2} \mathrm{O}_{2}$ for $30 \mathrm{~min}$ at room temperature, prior to being washed three times with PBS. After being blocked with 0.05\% Tween-20 (Shanghai Ling Feng Chemical Reagent Co., Ltd.) and 5\% bovine serum albumin (Gibco; Thermo Fisher Scientific, Inc., Waltham, MA, USA) in PBS for $30 \mathrm{~min}$ at room temperature, the slides were incubated with rabbit anti-FAM172A polyclonal antibody (cat. no. ab121364; Abcam, Cambridge, MA, USA) at a dilution of 1:200 at $4^{\circ} \mathrm{C}$ overnight. The slides were then washed with PBS and incubated with goat anti-rabbit IgG peroxidase-conjugated antibody (1:1,000; cat. no. bs10350; Bioword Technology, Inc., St. Louis Park, MN, USA) for $1 \mathrm{~h}$ at $37^{\circ} \mathrm{C}$. Following further washing with PBS, the slides were stained with DAB and were visualized using fluorescence microscopy (Axio Scope.A1; Zeiss, Oberkochen, Germany).

Cell culture and treatment. The IHH-4 human PTC cell line was provided by Professor Haixia Guan (First Hospital of China Medical University, Shenyang, China) and cultured in RPMI-1640 medium (Gibco; Thermo Fisher Scientific, Inc.) and Dulbecco's modified Eagle's medium (DMEM; Gibco; Thermo Fisher Scientific, Inc.) supplemented with $10 \%$ fetal bovine serum (Gibco; Thermo Fisher Scientific, Inc.), $100 \mathrm{U} / \mathrm{ml}$ penicillin and $0.1 \mathrm{mg} / \mathrm{ml}$ streptomycin (Gibco; Thermo Fisher Scientific, Inc.) in a $37^{\circ} \mathrm{C}, 5 \% \mathrm{CO}_{2}$ incubator. The medium was refreshed every $48 \mathrm{~h}$, and the cells were sub-cultured upon, or seeded into plates, on reaching $80 \%$ confluence. Subsequently, control PDC315 or eukaryotic expression vector PDC315-FAM172A plasmids were transfected into the cells using Lipofectamine 2000 (Invitrogen; Thermo Fisher Scientific, Inc.), according to the manufacturer's protocol. In the investigation of the role of the p38 MAPK pathway in FAM172A-stimulated proliferation, the IHH-4 cells were pre-incubated at $37^{\circ} \mathrm{C}$ for $2 \mathrm{~h}$ in dimethyl sulfoxide (DMSO; control) or $20 \mu \mathrm{M}$ SB202190 (dissolved in DMSO; Cell Signaling Technology, Inc., Danvers, MA, USA), a selective inhibitor of p38 MAPK, prior to being transferred with the plasmids.

MTT assay. An MTT assay was used to determine IHH-4 cell proliferation and was performed, as described previously $(11,12)$. In brief, the IHH-4 cells $\left(10^{4}\right.$ cells/well) were cultured in 96-well plates and treated, as described above. Following treatment $10 \mu \mathrm{l}$ MTT (Beyotime Institute of Biotechnology, Haimen, China) was added to each well for $4 \mathrm{~h}$ incubation at $37^{\circ} \mathrm{C}$. Following incubation, the medium was discarded and $150 \mu \mathrm{l}$ DMSO was added to each well and incubated for $5 \mathrm{~min}$ to dissolve the purple-blue formazan precipitate. Subsequently, the optical density (OD) was measured using an absorbance microplate reader (ELX800; Bio-Tek Instruments, Inc., Winooski, VT, USA) at a wavelength of $490 \mathrm{~nm}$. The data were obtained from each experiment with six replicates.

Cell growth curve. The IHH-4 cells were seeded into 24-well plates at $5 \times 10^{4}$ cells per well and transfected in accordance with methods as described above. Following intervention for 24, 48, 72 and $96 \mathrm{~h}$, respectively, cell counting was performed and a cell growth curve was constructed. Cell counting was performed using a hemocytometer (Shanghai Qiujing Biochemical Reagents Instrument Co., Ltd., Shanghai, China) under an XDS-1B model microscope (Chongqing Mike Photoelectric Instruments Co., Ltd., Chongqing, China).

Western blot analysis. The thyroid specimen proteins were extracted following being ground in liquid nitrogen, lysed with cell lysis buffer, containing radioimmunoprecipitation assay and phenylmethylsulfonyl fluoride (Beyotime Institute of Biotechnology), and centrifuged at $12,000 \mathrm{x} \mathrm{g}$ for $20 \mathrm{~min}$ at $4^{\circ} \mathrm{C}$. The cell proteins were collected and the extracted protein $(50 \mu \mathrm{g})$ was boiled for $5 \mathrm{~min}$ for denaturing and separated by $10 \%$ SDS-polyacrylamide gel electrophoresis (Beyotime Institute of Biotechnology) and then transferred onto a polyvinylidene fluoride membrane (Pall Corporation, Port Washington, NY, USA). Following blocking with 5\% non-fat milk in $1 X$ Tris-buffered saline with Tween 20 (Shanghai Ling Feng Chemical Reagent Co., Ltd.) for $1 \mathrm{~h}$ at room temperature, the membrane was incubated with primary rabbit antibodies against FAM172A (1:500; cat. no. ab121364; Abcam), GAPDH (1:1,000; cat. no. ap0063; Bioworld Technology, Inc., St. Louis Park, MN, USA) and antibodies associated with cell proliferation pathways, including p85 PI3K (1:1,000; cat. no. 4292), phosphorylated (p-)p85 PI3K (Tyr458; 1:1,000; cat. no. 4228), p38 MAPK (1:1,000; cat. no. 9212), p-p38 MAPK (Thr180/Tyr182; 1:1,000; cat. no. 9211), AMPK $\alpha$ (1:1,000; cat. no. 2532) and p-AMPK $\alpha$ (Thr172; 1:1,000; cat. no. 2535) from Cell Signaling Technology, Inc., at $4^{\circ} \mathrm{C}$ overnight. Subsequently, the membranes were incubated with anti-rabbit horseradish peroxidase (HRP)-conjugated IgG secondary antibody (1:5,000; cat. no. bs10350; Bioworld Technology, Inc.) for $1 \mathrm{~h}$ at room temperature. The protein bands were visualized using an enhanced chemiluminescence kit (Pierce Biotechnology, Inc., Rockford, IL, ISA) using Image Quant LAS 4000 mini chemiluminescence (GE Healthcare Bio-Sciences, Pittsburgh, PA, USA), and quantified using Gel-Pro Analyzer 4.0 software (Media Cybernetics, Inc., Rockville, MD, USA).

Statistical analysis. Each experiment was repeated at least three times and the results are expressed as the mean \pm standard deviation. One-way analysis of variance was used to 
analyze data using SPSS 19.0 software (IBM SPSS, Armonk, NY, USA). $\mathrm{P}<0.05$ (two-sided) was considered to indicate a statistically significant difference.

\section{Results}

Protein expression levels of FAM172A are increased in human PTC tissues. As shown in Fig. 1, the expression levels of FAM172A among the human normal thyroid, thyroid adenoma and PTC tissues were examined. The results of the immunohistochemical staining and western blotting demonstrated that the protein expression of FAM172A was highest in the human PTC tissues, compared with the normal thyroid and thyroid adenoma tissues $(\mathrm{P}<0.001$; Fig. $1 \mathrm{~A}$ and $\mathrm{B})$. Furthermore, in the patients with PTC, the protein expression level of FAM172A in the PTC tissues was significantly higher than that observed in the noncancerous tissues adjacent to the carcinoma in the same patient $(\mathrm{P}=0.001$; Fig. $1 \mathrm{C}$ and $\mathrm{D})$.

Overexpression of FAM172A accelerates $\mathrm{IHH}-4$ cell proliferation. To evaluate the proliferative effect of FAM172A on IHH- 4 cells, the numbers of cells were evaluated following transfection of the cells with PDC315-FAM172A or PDC315 plasmids using MTT assays and constructing a cell growth curve. As shown in Fig. 2A, the OD measured for the cells in the FAM172A group (1.09) was significantly higher than the OD of the control group (0.87; $\mathrm{P}=0.001)$. As shown in Fig. 2B, the cell growth curve indicated that the numbers of IHH-4 cells $\left(\times 10^{4}\right)$ in the group overexpressing FAM172A were $6.64 \pm 0.53,8.71 \pm 0.13,25.59 \pm 0.74$ and $44.96 \pm 0.94$ at $24,48,72$ and $96 \mathrm{~h}$, respectively, which were significantly higher than those in the control group of $5.73 \pm 0.18,7.38 \pm 0.35,17.41 \pm 0.29$ and $35.69 \pm 0.51$ at $24,48,72$ and $96 \mathrm{~h}$, respectively, at each time period $(\mathrm{P}=0.048$ at $24 \mathrm{~h} ; \mathrm{P}=0.004$ at $48 \mathrm{~h} ; \mathrm{P}<0.001$ at $72 \mathrm{~h}$ and $\mathrm{P}<0.001$ at 96 h; Fig. 2B).

P38 MAPK pathway is involved in the pro-proliferative effects of overexpressed FAM172A on IHH-4 cells. When FAM172A was transiently transfected into the IHH-4 cells for $24 \mathrm{~h}$, the overexpression of FAM172A was observed to trigger robust phosphorylation of p38 MAPK activation, however, no effect on the PI3K or AMPK signaling pathways were observed. As shown in Fig. 3A, the expression of p-p38 MAPK was prominently upregulated in the FAM172A group, compared with the expression in the control group, whereas no effect was observed on the expression of total p38 MAPK. No activation of PI3K or AMPK was observed. As shown in Fig. 3B and C, compared with the control group, the FAM172A-transfected group exhibited no significant difference in the protein expression levels of p-p85 PI3K or p-AMPK, and no changes were observed in the total protein levels of p85 PI3K and AMPK.

In order to further clarify the role of p38 MAPK on the proliferation of IHH- 4 cells induced by FAM172A, the inhibitor of p38 MAPK was used (SB202190). As shown in Fig. 3D, the activation of p38 MAPK, which was induced by FAM172A in the IHH-4 cells, was inhibited by SB202190. Correspondingly, the MTT assays demonstrated that FAM172A transfection increased the OD of the IHH- 4 cells by $23.4 \%$, compared with the cells in the control group $(\mathrm{P}<0.001)$, however, the

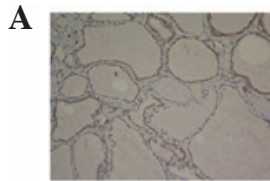

Normal thyroid

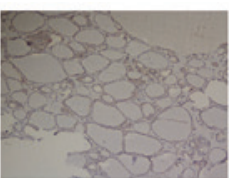

Thyroid adenoma Palliary thyroid carcinoma

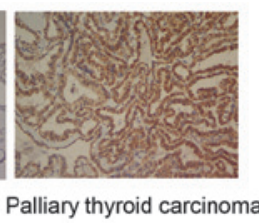

B
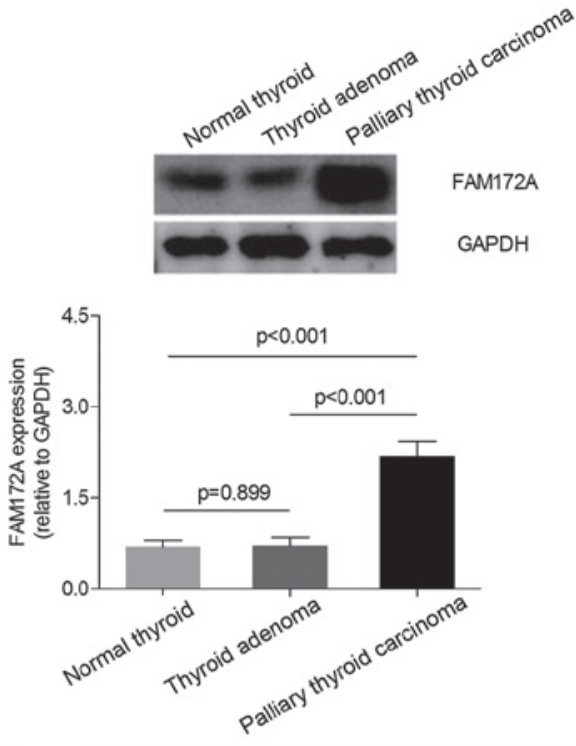

C

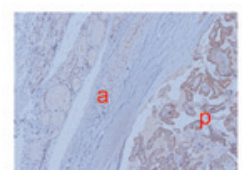

Palliary thyroid carcinoma 1

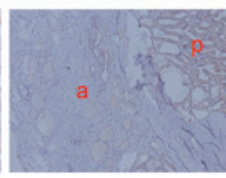

Palliary thyroid carcinoma 2

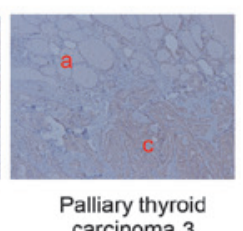

D
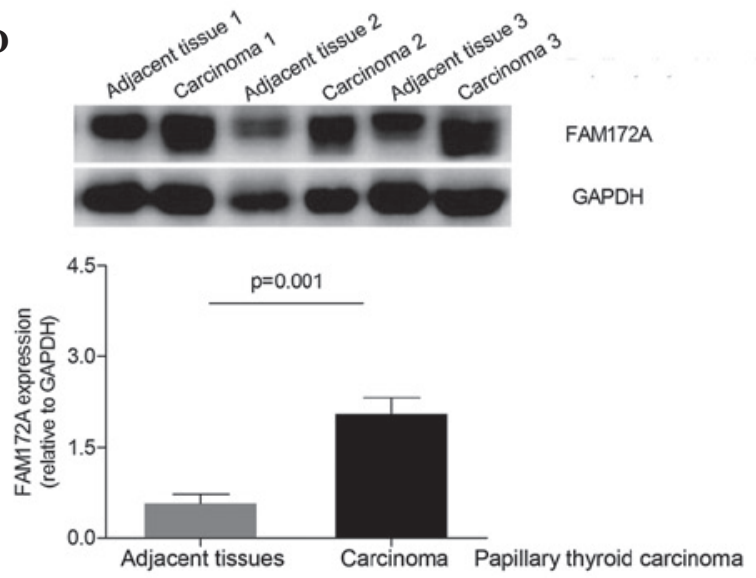

Figure 1. Comparison of the protein expression levels of FAM172A in human thyroid tissues. (A) Comparison of the protein expression levels of FAM172A among human normal thyroid tissues, thyroid adenoma tissues and papillary thyroid carcinoma tissues using immunohistochemical analysis (magnification, x10). (B) Comparison of the protein expression levels of FAM172A among the human normal thyroid tissues, thyroid adenoma tissues and papillary thyroid carcinoma tissues using western blot analysis $(\mathrm{P}<0.001)$. (C) Comparison of the protein expression levels of FAM172A between human papillary thyroid carcinoma tissues and adjacent tissues using immunohistochemical analysis. a, tissues adjacent to papillary thyroid carcinoma tissue. p, papillary thyroid carcinoma tissue. The numbers 1,2 and 3 represent three patients with PTC (magnification, x10). (D) Comparison of the protein expression levels of FAM172A between human papillary thyroid carcinoma tissues and adjacent tissues using western blot analysis $(\mathrm{P}<0.001)$. Data are expressed as the mean \pm standard deviation. FAM172A, family with sequence similarity 172 member A. 
A

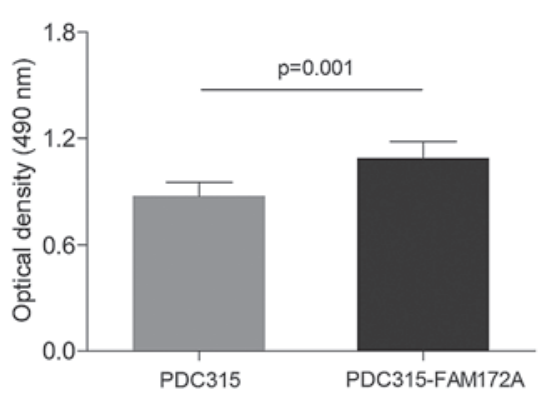

B

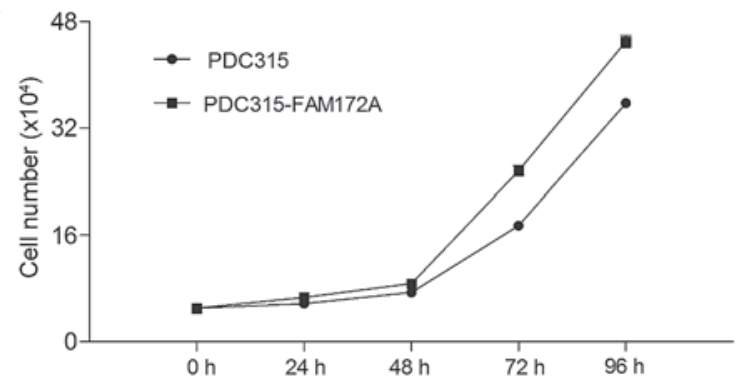

Figure 2. Overexpression of FAM172A markedly increases the rate of IHH-4 cell proliferation. (A) Optical density of the FAM172A group (1.09 \pm 0.09 ) was significantly higher, compared with that of the control group $(0.87 \pm 0.08 ; \mathrm{P}=0.001)$. (B) As the time following transfection of the IHH- 4 cells to overexpress FAM172A, the numbers of IHH-4 cells in the overexpressed FAM172A group were markedly higher, compared with those in the control group at $24 \mathrm{~h}$ $(\mathrm{P}=0.048), 48 \mathrm{~h}(\mathrm{P}=0.004), 72 \mathrm{~h}(\mathrm{P}<0.001)$ and $96 \mathrm{~h}(\mathrm{P}<0.001)$. Data are expressed as the mean \pm standard deviation. FAM172A, family with sequence similarity 172 , member A.

A

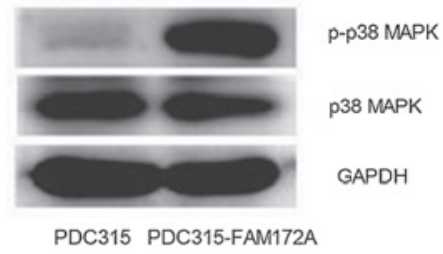

D

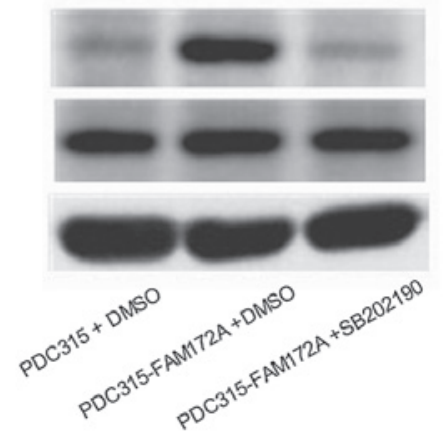

B

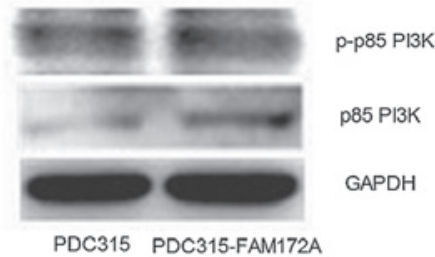

C

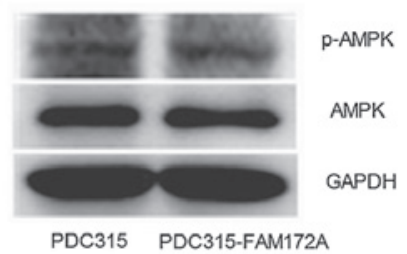

p-p38 MAPK

$\mathbf{E}$

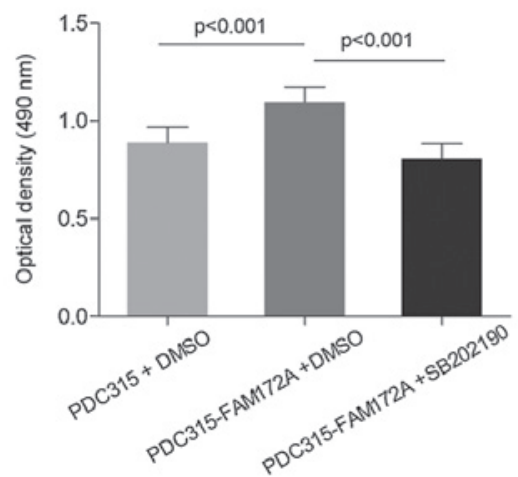

Figure 3. P38 MAPK pathway is involved in FAM172A-induced cell proliferation in IHH-4 cells. The effects of the overexpression of FAM172A on the protein expression levels of the (A) p38 MAPK, (B) PI3K and (C) AMPK pathways in the IHH-4 cells were examined. (D) Effects of SB202190, a selective p38 MAPK inhibitor, on FAM172A overexpression-induced activation of the p-p38 MAPK pathway in IHH-4 cells were examined. (E) Effects of SB202190 on FAM172A overexpression-induced proliferation in IHH-4 cells were examined. Data are expressed as the mean \pm standard deviation. FAM172A, family with sequence similarity 172, member A; PI3K phosphoinositide 3-kinase; MAPK, mitogen-activated protein kinase; AMPK, AMP-activated protein kinase; p-, phosphorylated; DMSO, dimethyl sulfoxide.

pro-proliferative effect of FAM172A on the IHH4 cells was significantly attenuated by SB202190 ( $\mathrm{P}<0.001$; Fig. 3E).

\section{Discussion}

In the present study, the protein expression levels of FAM172A in PTC were investigation, and it was found that the protein expression levels of FAM172A in PTC tissues were not only significantly higher than those in the noncancerous tissues adjacent to the carcinoma tissues, but they were also markedly higher than those in the normal thyroid and thyroid adenoma tissues. To the best of our knowledge, the present study is the first to confirm that high protein expression levels of FAM172A are found in human PTC.

PTC is the most common type of malignant tumor of the endocrine system, and statistics have shown that its incidence has continuously and sharply increased over previous decades worldwide $(13,14)$. A previous study indicated that thyroid cancer is the fifth most common type of cancer in women (15) and, in Italy, thyroid cancer is the second most frequent type of cancer in women $<45$ years of age (16). Therefore, early detection and prevention is of crucial importance for thyroid cancer. Various examination techniques, including thyroid ultrasound, radionuclide imaging and fine needle aspiration cytology have the potential to improve the accuracy of the diagnosis of thyroid cancer $(17,18)$, however, there remains a lack of simple and effective methods to enable early differential diagnoses for benign and malignant thyroid nodules. In our previous study, it was demonstrated that there is a signal peptide sequence in the $\mathrm{N}$ paragraph of the FAM172A protein, determined using bioinformatics techniques (3), and this signal peptide is a noteworthy feature of the secreted protein (19). 
Considering that uncontrolled cell growth has been widely known as a fundamental factor for tumor occurrence and progression $(20,21)$, human PTC IHH-4 cells were used as a model of PTC in the present study to further examine the underlying role of the FAM172A protein in PTC. A noticeable increase $(25 \%)$ in the OD of the IHH- 4 cells was observed following transfection of the cells with the FAM172 overexpression vectors. The cell growth curve also showed the same results, and these results suggested that FAM172A may be important in the pathogenesis of PTC. Analogous to the results of the present study, our previous study demonstrated that HEK293 cells transfected with PDC315-FAM172A vectors proliferated at a markedly faster rate, compared with those transfected with PDC315 vectors (1).

Subsequently, the present study investigated the effect of FAM172A on several common signaling pathways associated with cell proliferation, including the PI3K, p38 MAPK and AMPK signaling pathways, in IHH-4 cells (22-24). The results demonstrated that the overexpression of FAM172A caused marked activation of the p38 MAPK pathway, and this pathway activation was inhibited following treatment with SB202190, a selective inhibitor of p38 MAPK. Correspondingly, FAM172A-induced cell proliferation was attenuated following treatment with SB202190. The above results indicated that FAM172A may promote cell proliferation via the p38 MAPK pathway and, thus be involved in the disease course of PTC. Consistent with these results, a study by Pomérance et al (25) showed that p-p38-MAPK is markedly expressed in PTC cells; Huang et al (26) reported that norepinephrine stimulates pancreatic cancer cell proliferation through activation of the p38 MAPK pathway, and Ayllón V et al (27) demonstrated that PBK/TOPK promotes tumour cell proliferation through the p38 MAPK activity. However, Gao et al (28) reported that the p38 MAPK pathway is involved in the pro-apoptotic effect of notoginsenoside Ft1 on SH-SY5Y human neuroblastoma cells. The different results among these previous studies may be due to p38 MAPK having have multiple functions, including cell proliferation and apoptosis, which appear to act differently depending on the types of cells and the specific conditions (29).

The present study has potential implications regarding the pathogenesis and treatment of PTC. It was suggested that FAM172A-p38 may be associated with the mechanisms underlying the evolution and progression of PTC, since it was demonstrated that FAM172A was able to activate the p38 signaling pathway, thus promoting cell proliferation, which is an important mechanism underlying tumor progression. In addition, FAM172A may be used to identify benign and malignant thyroid tumors, as increased expression of FAM172A was detected in PTC. The results of the present study suggested that FAM172A and p38 may be used as potential drug targets for the adjuvant treatment of PTC, since FAM172A may activate the p38 signaling pathway and promote cell proliferation.

In conclusion, the present study was the first, to the best of our knowledge, to verify significantly high protein expression levels of FAM172A in human PTC. The present study also presented evidence that FAM172A may promote cell proliferation via activating p38 MAPK signaling pathway and, therefore, be involved in the pathogenesis of PTC. The results of the present study extends current knowledge of FAM172A, a novel protein, and its role in disease states, and supports its use as a novel and simple biomarker for the early identification of PTC.

\section{Acknowledgements}

The authors would like to thank the Department of Surgery of Shanghai Jiao Tong University Affiliated Sixth People's Hospital for providing the human thyroid tissue samples, and would also like to thank Professor Haixia Guan for provision of the IHH-4 human PTC cell line. This study was supported by grants from the National Natural Science Foundation of China (grant. nos. 81170759 and 81502316), the Shanghai Science and Technology Commission Funded Project (grant. no. 14411964100) and the Shanghai Scientific Research Innovation Projects (grant. no. 1322015).

\section{References}

1. Li LX, Zhou WB, Tao Z, Deng WJ, Liang WC, Yang ZH, Ye WW, Bao YQ, Jia WP and Hu RM: Effect of FAM172A protein on apoptosis and proliferation in HEK293 cells. Zhonghua Yi Xue Za Zhi 90: 2424-2427, 2010 (In Chinese)

2. Li L, Dong X, Leong MC, Zhou W, Yang Z, Chen F, Bao Y, Jia W and Hu R: Identification of the novel protein FAM172A and its up-regulation by high glucose in human aortic smooth muscle cells. Int J Mol Med 26: 483-490, 2010.

3. Li LX, Tao Z, Dong XH, Liang WC, Yang ZH, Mou B, Bao YQ, Wang C, Jia WP and Hu RM: Molecular cloning of a novel gene, C5orf21 gene and its roles in diabetic macroangiopathy. Zhonghua Yi Xue Za Zhi 89: 2574-2577, 2009 (In Chinese).

4. Harding JL, Shaw JE, Peeters A, Cartensen B and Magliano DJ: Cancer risk among people with type 1 and type 2 diabetes: Disentangling true associations, detection bias and reverse causation. Diabetes Care 38: 734-735, 2015.

5. Ayturk S, Gursoy A, Kut A, Anil C, Nar A and Tutuncu NB: Metabolic syndrome and its components are associated with increased thyroid volume and nodule prevalence in a mild-to-moderate iodine-deficient area. Eur J Endocrinol 161: 599-605, 2009.

6. Gursoy A: Rising thyroid cancer incidence in the world might be related to insulin resistance. Med Hypotheses 74: 35-36, 2010.

7. Bae MJ, Kim SS, Kim WJ, Yi YS, Jeon YK, Kim BH, Lee BJ, Lee JC, Kim IJ, Wang SG and Kim YK: High prevalence of papillary thyroid cancer in Korean women with insulin resistance. Head Neck 8. doi: 10.1002/hed.23848, 2014.

8. Guo K and Wang Z: Risk factors influencing the recurrence of papillary thyroid carcinoma: A systematic review and meta-analysis. Int J Clin Exp Pathol 7: 5393-5403, 2014.

9. Li Y, Nakamura M and Kakudo K: Targeting of the BRAF gene in papillary thyroid carcinoma (review). Oncol Rep 22: 671-681, 2009.

10. Krawczyk-Rusiecka K, Wojciechowska-Durczyńska K, Cyniak-Magierska A, et al: COX-2 expression in papillary thyroid carcinoma (PTC) in cytological material obtained by fine needle aspiration biopsy (FNAB). Thyroid Res 4:3, 2011.

11. van Meerloo J, Kaspers GJ and Cloos J: Cell sensitivity assays: The MTT assay. Methods Mol Biol 731: 237-245, 2011.

12. Lee SB, Li DQ, Tan DT, Meller DC and Tseng SC: Suppression of TGF-beta signaling in both normal conjunctival fibroblasts and pterygial body fibroblasts by amniotic membrane. Curr Eye Res 20: 325-334, 2000.

13. Schlumberger MJ: Papillary and follicular thyroid carcinoma. N Engl J Med 338: 297-306, 1998.

14. LiVolsi VA: Papillary thyroid carcinoma: An update. Mod Pathol 24 (Suppl 2): S1-S9, 2011.

15. Siegel R, Naishadham D and Jemal A: Cancer statistics, 2012. CA Cancer J Clin 62: 10-29, 2012. 
16. Dal Maso L, Lise M, Zambon P, Falcini F, Crocetti E, Serraino D, Cirilli C, Zanetti R, Vercelli M, Ferretti S, et al: Incidence of thyroid cancer in Italy, 1991-2005: Time trends and age-period-cohort effects. Ann Oncol 22: 957-963, 2011.

17. Multanen M, Haapiainen R, Leppäniemi A, Voutilainen P and Sivula A: The value of ultrasound-guided fine-needle aspiration biopsy (FNAB) and frozen section examination (FS) in the diagnosis of thyroid cancer. Ann Chir Gynaecol 88: 132-135, 1999.

18. Naswa N, Sharma P, Suman Kc S, Lata S, Kumar R, Malhotra A and Bal C: Prospective evaluation of 68Ga-DOTA-NOC PET-CT in patients with recurrent medullary thyroid carcinoma: Comparison with 18F-FDG PET-CT. Nucl Med Commun 33 766-774, 2012

19. Joly DL, Feau N, Tanguay P and Hamelin RC: Comparative analysis of secreted protein evolution using expressed sequence tags from four poplar leaf rusts (Melampsora spp.). BMC Genomics 11: 422, 2010.

20. Evan GI and Vousden KH: Proliferation, cell cycle and apoptosis in cancer. Nature 411: 342-348, 2001.

21. López-Sáez JF, de la Torre C,Pincheira J and Giménez-Martín G: Cell proliferation and cancer. Histol Histopathol 13: 1197-1214, 1998.

22. Qu JL, Qu XJ, Zhao MF, Teng YE, Zhang Y, Hou KZ, Jiang YH, Yang XH and Liu YP: Gastric cancer exosomes promote tumour cell proliferation through PI3K/Akt and MAPK/ERK activation. Dig Liver Dis 41: 875-880, 2009.
23. Mihaylova MM and Shaw RJ: The AMPK signalling pathway coordinates cell growth, autophagy and metabolism. Nat Cell Biol 13: 1016-1023, 2011.

24. Hardwick JC, van den Brink GR, Offerhaus GJ, van Deventer SJ and Peppelenbosch MP: NF-kappaB, p38 MAPK and JNK are highly expressed and active in the stroma of human colonic adenomatous polyps. Oncogene 20: 819-827, 2001.

25. Pomérance M, Quillard J, Chantoux F, Young J and Blondeau JP: High-level expression, activation and subcellular localization of p38-MAP kinase in thyroid neoplasms. J Pathol 209: 298-306, 2006.

26. Huang XY, Wang HC, Yuan Z, Huang J and Zheng Q: Norepinephrine stimulates pancreatic cancer cell proliferation, migration and invasion via $\beta$-adrenergic receptor-dependent activation of P38/MAPK pathway. Hepatogastroenterology 59: 889-893, 2012.

27. Ayllón V and O'connor R: PBK/TOPK promotes tumour cell proliferation through p38 MAPK activity and regulation of the DNA damage response. Oncogene 26: 3451-3461, 2007.

28. Gao B, Shi HL, Li X, Qiu SP, Wu H, Zhang BB, Wu XJ and Wang ZT: P38 MAPK and ERK1/2 pathways are involved in the pro-apoptotic effect of notoginsenoside Ft1 on human neuroblastoma SH-SY5Y cells. Life Sci 108: 63-70, 2014.

29. Cuadrado A and Nebreda AR: Mechanisms and functions of p38 MAPK signaling. Biochem J 429: 403-417, 2010. 